\title{
Modelling the vertical UL 94 test: competition and collaboration between melt dripping, gasification and combustion
}

\author{
Florian Kempel ${ }^{1}$, Bernhard Schartel ${ }^{1, *,}$, Julio M. Marti $^{2}$, Kathryn M. Butler ${ }^{3}$, \\ Riccardo Rossi ${ }^{2}$, Sergio R. Idelsohn ${ }^{2,4}$, Eugenio Oñate ${ }^{2}$ and Anja Hofmann ${ }^{1}$ \\ ${ }^{1}$ BAM Federal Institute for Materials Research and Testing, Unter den Eichen 87, 12205 Berlin, Germany \\ ${ }^{2}$ International Center for Numerical Methods in Engineering (CIMNE), Building C1, Campus North UPC, C/ Gran \\ Capita s/n, 08034 Barcelona, Spain \\ ${ }^{3}$ National Institute of Standards and Technology (NIST), Fire Research Division, 100 Bureau Drive, Stop 8665, \\ Gaithersburg, MD 20899-8665, USA \\ ${ }^{4}$ Catalan Institution for Research and Advanced Studies (ICREA), Passeig Lluís Companys, 23, 08010 Barcelona, Spain
}

\begin{abstract}
SUMMARY
An experimental and numerical investigation of the effect of bisphenol A bis(diphenyl phosphate) (BDP) and polytetrafluoroethylene (PTFE) on the fire behaviour of bisphenol A polycarbonate/acrylonitrile butadiene styrene (PC/ABS) in the vertical UL 94 scenario is presented. Four PC/ABS blends were discussed, which satisfy different UL 94 classifi cations d ue to the competing ef fects of gasifica ti on, charring, flame inhibition and melt flow/dripping. For numerical investigation, the particle finite element method (PFEM) is used. Its capability to model the complex fire behaviour of polymers in the UL 94 is analysed. The materials' properties are characterised, in particular the additives impact on the dripping behaviour during thermal exposure. BDP is an efficie nt $\mathrm{p}$ lasticiser; adding PTFE $\mathrm{p}$ reve nts dripping by causing a flo $\mathrm{w}$ limit. P FEM simulation $\mathrm{s}$ reproduce the dripping and burning behaviour, in particular the competition between gasification and dripping. The thermal im- pact of both the burner and the flame is approximated taking into account flame inhibition, charring and effective heat of combustion. PFEM is a promising numerical tool for the investigation of the fire behaviour of polymers, particularly when large deformations are involved. Not only the principal phenomena but also the different UL 94 classi fi cations and the exti nc tion times are well predicted.
\end{abstract}

KEY WORDS: melt dripping; UL 94; particle finite element method (PFEM); simulation; bisphenol A polycarbonate/acrylonitrile butadiene styrene (PC/ABS); polytetrafluoroethylene (PTFE); bisphenol A bis(diphenyl phosphate) (BDP)

Polymer materials, in particular thermoplastics, easily lose their dimensional stability when their glass transition or melting temperature, respectively, is exceeded. At elevated temperatures close to or equalling pyrolysis temperature, most thermoplastics tend to exhibit extensive melt flow and dripping. In a fire, these effects can have different consequences. On the one hand, melt flow and/or dripping of burning material often lead to higher burning rates (e.g. due to larger surface area), additional pool fires on the ground or enhanced downward flame spread. On the other hand, fuel and heat are removed from the actual pyrolysis front or fire source by dripping or melt flow. Reducing the amount of material involved in the actual pyrolysis can prevent ignition, slow down the fire growth or result in extinction [1-5].

The vertical UL 94 test (IEC 60695-11-10) is an important small-scale fire test used to assess the flammability of polymer materials $[6,7]$. The effects of melt flow and dripping are considered in the UL 
94 test and to some extent crucial in deciding on the final classification. Flaming drips lower the UL 94 classification towards $\mathrm{HB}$ or $\mathrm{V}-2$, while extinction by nonflaming dripping is one efficient way to achieve the desired V-1 or V-0 classification. Thus, apart from gasification of fuel, charring, effective heat of combustion of the volatiles and flame inhibition, the melt flow and dripping are the most important phenomena determining the fire behaviour of thermoplastics in the vertical UL 94 scenario. It is quite a challenge in the UL 94 test to achieve a V-0 or V-1 rating, a widely used definition for flame retarded polymeric materials and a common requirement in electrical engineering and electronic applications. Nevertheless, as the test merely provides a classification, it is often of limited use for further optimisation and future development. Differentiated assessment and mechanistic aspects of burning are not considered. In the literature, some studies deal with the quantitative analysis of the testing conditions or the materials' behaviour in the UL 94 test, but more detailed or modelling approaches are rare [8-12].

Numerical simulations are used increasingly to analyse and predict flame spread and fire growth in different fire scenarios. Computational fluid dynamics are typically applied to solve and combine submodels for fluid flows, gas-phase combustion, heat transfer and condensed-phase decomposition. They are usually based on the Navier-Stokes equations and are discretised by dividing the domain into a fixed grid. This prevents the calculation of large deformations such as those that apply in the case of melt flow or dripping. The increasing interest in so-called fluid-structure interaction problems has led to the development of robust and efficient numerical methods for the analysis of engineering problems involving the interaction of fluids and structures. These methods account for large motions of the fluid-free surface and the existence of fully or partially submerged bodies. Recent studies by Butler et al. have shown the potential of these methods when applied to fire problems [13-15].

In the present study, the fire behaviour of four bisphenol A polycarbonate / acrylonitrile butadiene styrene (PC/ABS) blends in the UL 94 scenario is investigated experimentally and numerically. The
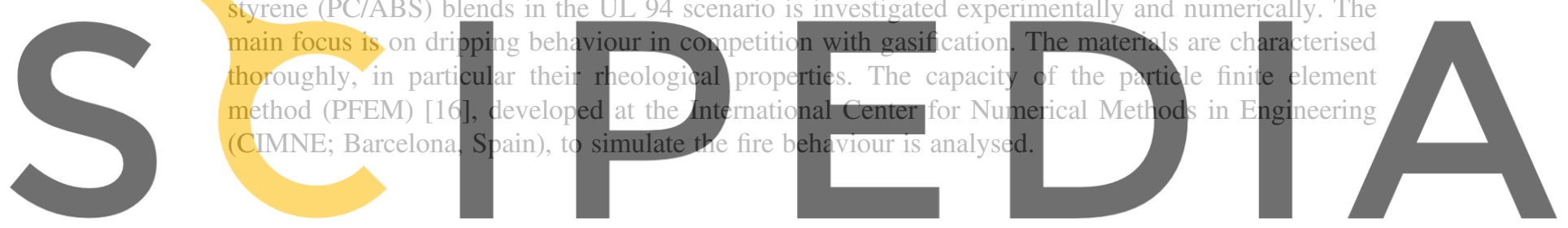

Register for free at https//www.scipedia.com to download the version without the watermark 2.1. Materials

The material system consists of four PC/ABS biends with and without the flame retardant bisphenol A bis (diphenyl phosphate) (BDP) and polytetrafluorethylene (PTFE) as an antidripping agent. The materials with BDP contain $12.5 \%$ mass fraction of the flame retardant. The materials with PTFE contain $0.9 \%$ mass fraction of a master batch consisting of styrene-acrylonitrile (SAN) and PTFE in a ratio of 1:1. The four different materials investigated are PC/ABS, PC/ABS + BDP, PC/ABS + PTFE and PC/ $\mathrm{ABS}+\mathrm{BDP}+\mathrm{PTFE}$ [17]. The materials were provided by Bayer MaterialScience AG (Dormagen, Germany). ${ }^{\ddagger}$ The blends were not commercial available, but the composition of PC/ABS and PC/ $\mathrm{ABS}+\mathrm{BDP}+\mathrm{PTFE}$ were quite close to commercial products. Thus, this study was targeting scientific understanding as well as application.

\subsection{Test procedures}

UL 94 tests were performed according to the Underwriters Laboratory test for the flammability of plastics (UL 94), IEC 60695-11-10. Specimens with the dimensions $(125 \times 13 \times 1.5) \mathrm{mm}$ were tested in the vertical orientation. Additionally, the typical mass loss of the samples during the test was determined in

\footnotetext{
${ }^{\ddagger}$ Certain commercial equipment, instruments or materials are identified in this paper in order to specify the experimental procedure adequately. Such identification is not intended to imply recommendation or endorsement by the National Institute of Standards and Technology, nor is it intended to imply that the materials or equipment identified are necessarily the best available for the purpose. The policy of NIST is to use metric units of measurement in all its publications and to provide statements of uncertainty for all original measurements. In this document, however, data from organizations outside NIST are shown, which may include measurements in nonmetric units or measurements without uncertainty statements.
} 
a separate series of experiments. For this, the sample holder was placed on a scale and the mass of the setup recorded during the UL 94 test. Dripping material was caught before touching the scale and hence considered as material lost.

Thermogravimetric analysis was conducted using a TGA/SDTA 851 from Mettler Toledo. The measurements were performed under nitrogen with a sample weight of $10 \mathrm{mg}$. Measurements for kinetic analysis were taken at heating rates of $(1,2,5$ and 10) $\mathrm{K} / \mathrm{min}$. Differential scanning calorimetry (DSC) was conducted with a DSC 7 from Perkin Elmer under a nitrogen atmosphere. The sample weight was between 2 and $4 \mathrm{mg}$, and the heating rate was $5 \mathrm{~K} / \mathrm{min}$.

For rheological investigations, the rheometer Anton Paar Physica MCR301 was used, with plate-plate geometry, a plate gap of $1 \mathrm{~mm}$ and a plate diameter of $25 \mathrm{~mm}$. Isothermal measurements were conducted at four temperatures $(488 \mathrm{~K}, 498 \mathrm{~K}, 543 \mathrm{~K}$ and $588 \mathrm{~K})$ in the frequency sweep mode. The frequency was varied between $100 \mathrm{~Hz}$ and $0.1 \mathrm{~Hz}$, and a small deformation $(\gamma=0.5 \%)$ was given. Since the temperatures chosen were clearly below the decomposition temperature, the measurements were recorded under air. One set of six PC/ABS samples was pretreated in a tube furnace under nitrogen. The materials were put in the furnace for $480 \mathrm{~s}$. For each sample, the furnace was set to a different temperature of $503 \mathrm{~K}, 633 \mathrm{~K}, 653 \mathrm{~K}, 673 \mathrm{~K}$ and $693 \mathrm{~K}$, respectively.

3. NUMERICAL SIMULATION TOOL

\subsection{The particle finite element method}

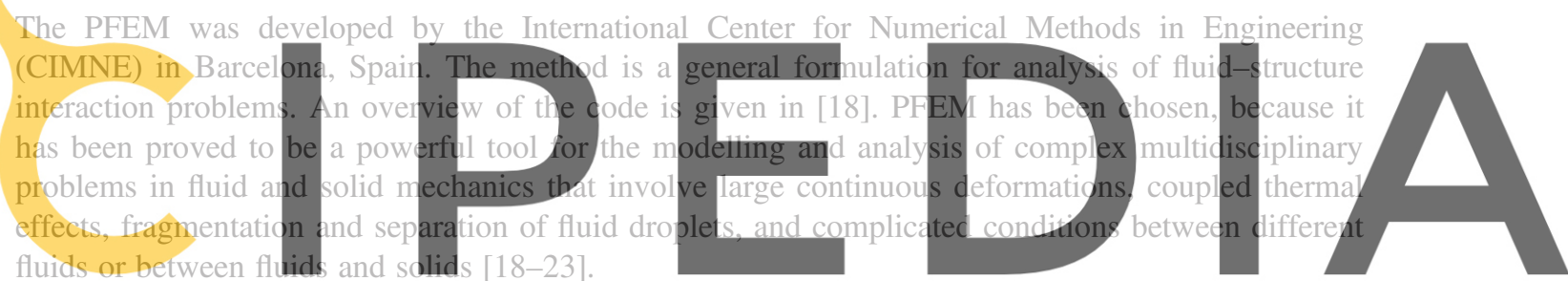
fluids or between fluids and sdids [18-23].

Particle finite element method combines particle-based techniques with the standard finite element

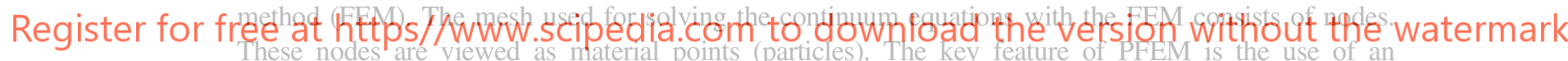

updated Lagrangian description to model the motion of nodes, which are tracked during the transient solution. According to their density, initial acceleration and velocity, and subject to the force of gravity, the nodes can move freely and can even separate from the main analysis domain, transporting their momentum and physical properties. Once the motion is applied, a robust and efficient remeshing algorithm connects the nodes into a finite element grid for solution of the state variables in the new configuration. The basic PFEM algorithm involves the following steps:

- Definition of particles (with a given velocity, density, gravity, etc.), volume, boundary conditions and free surfaces.

- Evaluation of the forces in the particles by solving the Navier-Stokes equations using the FEM. This requires generating the mesh, which is done using extended Delaunay tessellation [24].

- Evaluation of the velocity and acceleration of each particle using the updated Lagrangian description.

- Moving the particles and proceeding with step 1.

Regarding the analysis of problems related to polymeric materials, additional submodels are applicable, as described in [13]. In particular, the thermal decomposition (also gasification) of the materials is of major interest in simulating the fire behaviour of polymers. Additional terms in the conservation equations for mass and energy account for the mass loss as a result of gasification and the energy absorbed during gasification, respectively. The heat flux due to gasification is calculated based on the product of density, enthalpy of decomposition and the temperature-dependent volume variation. In the current version, thermal decomposition kinetics is expressed by a single-step, first-order Arrhenius equation. The validation of the gasification algorithm is shown in [15]. 


\subsection{Model setup}

The UL 94 setup was represented by a rectangular sample positioned in the vertical direction and exposed to an external heat flux. The dimensions correspond to the original samples. The UL 94 test is a complex scenario with respect to the quantification of heat transfer to the sample. High temperatures concentrated on a limited space introduce strong temperature gradients. Additionally, edge effects are of major influence [10], indicating the need for a spatial approach to determine the testing conditions. Apart from 3D simulations, this study also used 2D simulations to assess the capabilities of PFEM with reasonable computational cost. In 2D calculations, the sample was discretised by 1978 nodes forming 3701 threenoded triangular cells. In 3D calculations, 5727 nodes and 21,854 four-noded tetrahedra form the sample. Clamping was modelled by fixing the velocities of the nodes on the top of the sample. The computational domain consisted of a square or cube, respectively, with a side length of $300 \mathrm{~mm}$. The centre of the upper surface of the domain was aligned with the top of the sample. Dripping particles, which are accelerated due to the gravitational force, are followed until they reach the boundary of the domain. The atmosphere around the sample was not considered in the simulation. Time step for simulation was $0.01 \mathrm{~s}$.

In the vertical UL 94 test, a flame produced by a Bunsen burner is applied to the sample twice, each time for a duration of $10 \mathrm{~s}$. For simplification, the model setup concentrated on the sample's behaviour under two distinct conditions: during a constant external heat flux and during/after the ignition source had been applied once for $10 \mathrm{~s}$.

The heat flux distribution $(q(x))$ at the surface of the specimen is an important boundary condition. In the absence of better data, the heat flux distribution was estimated somewhat arbitrarily when igniting with the burner and for the flame of the burning material. Nevertheless, the estimations used are quite

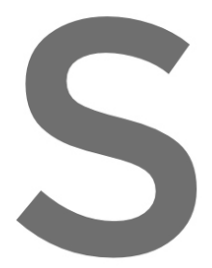
reasonable adjusting distance to the botto The heat flux distrib time step. In the sim sample was estimat characterised. The maxil the simulations during the ignition phase of the UL 94 test, when the burner is applied, the rate of heat flow to the sample due to the burner was set to $\sim 48 \mathrm{~W}\left(\max Q=150 \mathrm{~kW} / \mathrm{m}^{2}, q(x)\right.$ decreasing with the

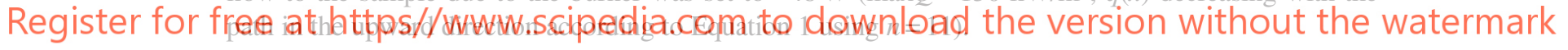

$$
q(x)=\frac{(125-x)^{n}}{125^{n}} * \max Q
$$

Additionally, heat was introduced to the sample as a result of polymer combustion and the heat feedback from the flame. Therefore, a simple, straightforward combustion model was used to estimate the impact of the flame, taking into account the main parameters controlling the combustion: The heat release rate of the sample was calculated by the product of the mass loss rate (simulated by PFEM), heat of combustion of the volatiles and the combustion efficiency. The heat of combustion of the volatiles $\left(h_{\text {cvol }}\right)$ and the combustion efficiency $(\chi)$ were experimental input parameters taken from literature [17]. The latter is one for the wellventilated UL 94 test and reduced when flame inhibition occurs. Thus, gas-phase flame retardancy mechanisms such as fuel dilution and flame inhibition were taken into account properly. In the current version of the PFEM code, no submodel for charring or residue formation was implemented. Thus, the calculated mass loss consisted not only of mass released but also mass transferred to char. To consider the main impact of charring, the heat release rate was reduced by the char yield $(\mu)$ of the blends obtained from experiments [17]. The heat release rate was converted into heat flux to the sample assuming that the heat flux maximum is present at the bottom of the sample, decreasing with the square of the path in the upward direction (Equation $1, n=2, \max Q=\Delta m h_{\mathrm{cvol}} \chi(1-\mu)$ ). The maximum heat flux to the sample at the bottom was limited to $\max Q=150 \mathrm{~kW} / \mathrm{m}^{2}$. The heat flux distribution was recalculated taking into account the deformation of the surface for each time step.

In PFEM, the nodes bear the material properties, as described above. For the numerical investigation, isothermal values are used for the density, specific heat capacity and thermal conductivity of the material. 
The viscosity was defined as a function of temperature. In the simulations, it is assumed that the polymer melt behaves like an incompressible fluid. A quasi-rigid behaviour of the polymer object at room temperature is reproduced by setting the viscosity to a sufficiently high value. This ensures that the unheated polymer moves a negligible distance over the duration of the problem. As temperature increases in the thermoplastic object due to heat exposure, the viscosity decreases by several orders of magnitude as a function of temperature. This induces the melting and flow of the particles in the heated zone. Numerically, the temperature-dependent viscosity is implemented by the standard constitutive equation for an incompressible Newtonian fluid. Generally polymer melts do not exhibit a linear shear stress versus strain rate curve and are known to behave like non-Newtonian fluids. But for higher temperatures and regarding a narrow range of shear rates like those experienced in the UL 94 test, the assumption of Newtonian behaviour becomes satisfactory [15].

\section{RESUI TS AND DISCUSSION}

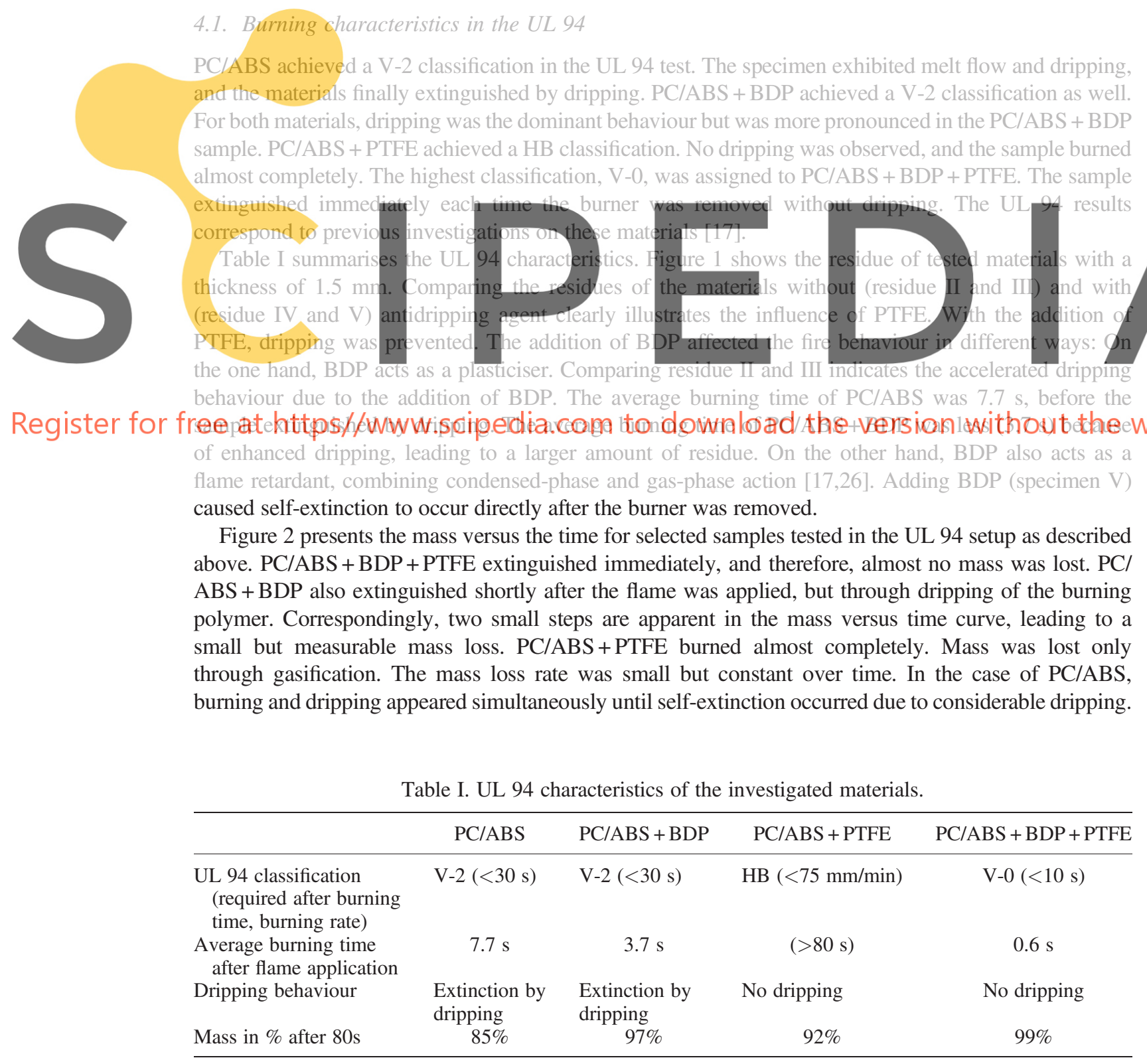




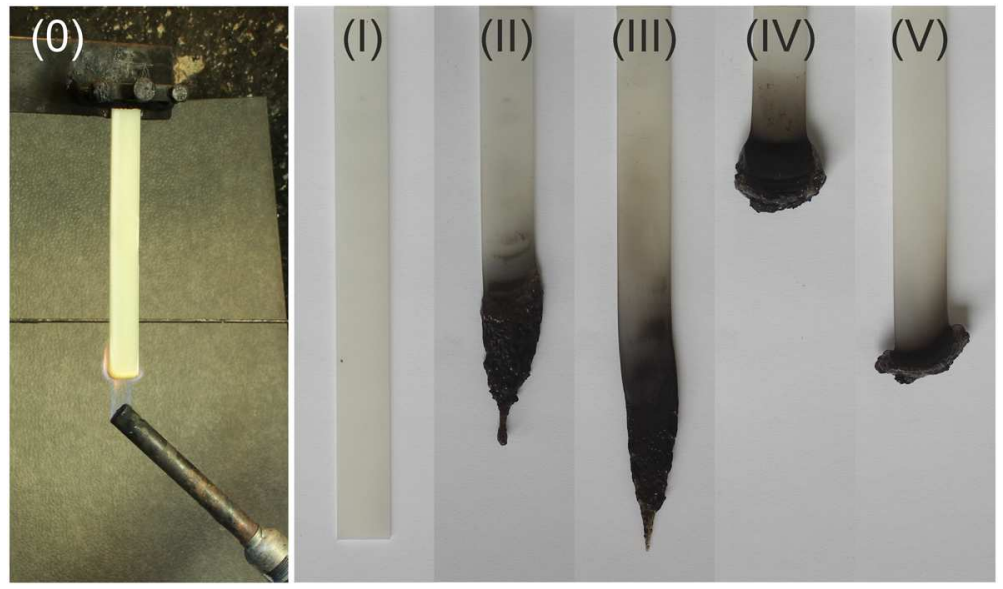

Figure 1. UL 94 vertical setup (0), original specimen (I) and UL 94 residues of PC/ABS (II), PC/ABS + BDP (III), PC/ABS + PTFE (IV) and PC/ABS + BDP + PTFE (V).
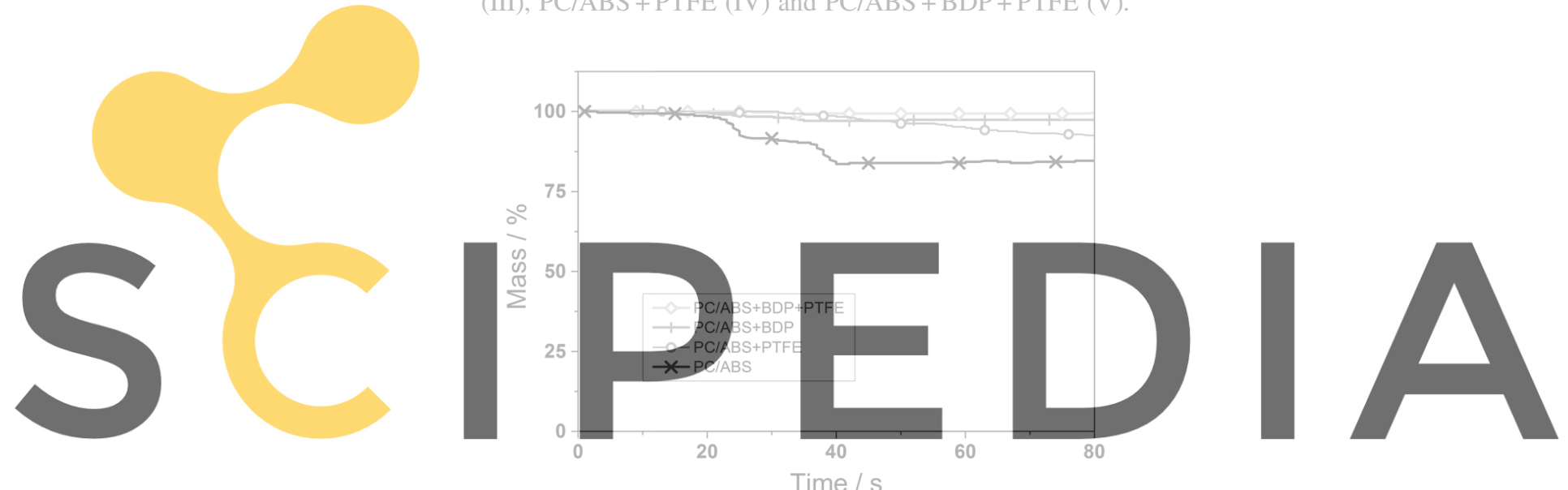

Register for free at https//www.scipedia.com to download the version without the watermark

For PC/ABS + PTFE samples, the mass loss rate as a result of thermal decomposition was determined. In the region of constant burning, the average mass loss rate of PC/ABS + PTFE samples with a thickness of $1.5 \mathrm{~mm}$ was $\sim 0.005 \pm 0.002 \mathrm{~g} / \mathrm{s}$. This value agrees with the mass loss rates of various materials in the vertical UL 94 test reported in [8].

\subsection{Rheology}

Melt flow and dripping occur as a result of the applied stresses and the rheological characteristics of a sample. In the following, the rheological characterisation of the materials is presented. The rheology was studied in order to understand and quantify the impact of the additives PTFE and BDP on the dripping behaviour of PC/ABS.

Figure 3 shows the shear stress versus the shear rate of the materials. The master curves with the reference temperature of $523 \mathrm{~K}$ were determined from isothermal measurements of the four materials. BDP is not only a flame retardant but also a very efficient plasticiser that is often desired to improve processability in such thermoplastic systems. The shear stress versus shear rate and thus viscosity of PC/ $\mathrm{ABS}+\mathrm{BDP}$ compared to PC/ABS as well as of PC/ABS + BDP + PTFE compared to PC/ABS + PTFE decrease by more than a factor of 4 . For the analysis of dripping behaviour, the region of low shear rates is of principal interest. Only in this region does the addition of PTFE influence the curve shape, considerably changing the melt flow and dripping behaviour without deteriorating the improved processability [27]. Without PTFE, the shear stress decreases with decreasing shear rates. The addition of PTFE leads to a flow limit, i.e. even at very low shear rates the sample shows a certain resistance to 


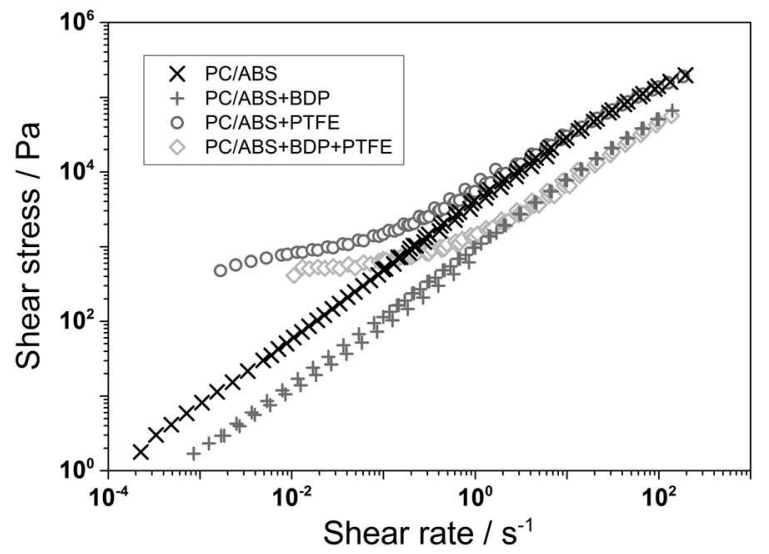

Figure 3. Master curves of shear stress versus shear rate with the reference temperature of $523 \mathrm{~K}$.

stresses. Translated to the material's behaviour in the UL 94 test, the flow limit of materials containing PTFE prevents the samples from dripping. In contrast, at elevated temperatures and in particular at low shear rates, materials without PTFE do not resist gravitational forces so that dripping takes place.

For the numerical investigation, the viscosity as a function of temperature is a key parameter to describe the melt flow and dripping behaviour. Generally, viscosity decreases with increasing temperatures, but thermal decomposition, too, may lead to a change in viscosity. In order to determine the influence of thermal decomposition on the rheology of PC/ABS, samples were pretreated in a tube furnace before testing in the rheomete. Figure 4 shows the materials after pretreatnent at various temperatures. The colouring indicates the different stages of decomposition.

The impact on rheology is seen in Figure 5. The viscosity as a function of shear rate measured at $588 \mathrm{~K}$ is shown. The sample pretreated slightly above melting temperature graph of the untreated one. Samples pretreated at higher temperatur Register for free at https//www.scipedia.com (II!) do download the version without the watermark
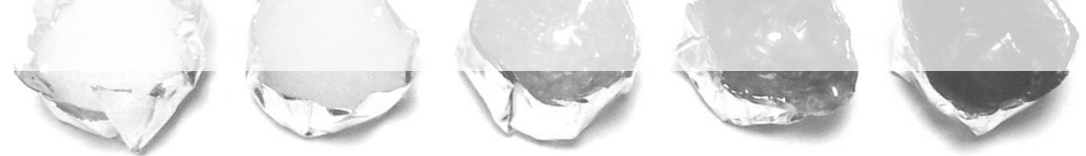

Figure 4. PC/ABS samples pretreated for $480 \mathrm{~s}$ in a tube furnace at (I) $503 \mathrm{~K}$, (II) $633 \mathrm{~K}$, (III) $653 \mathrm{~K}$, (IV) $673 \mathrm{~K}$ and (V) $693 \mathrm{~K}$.

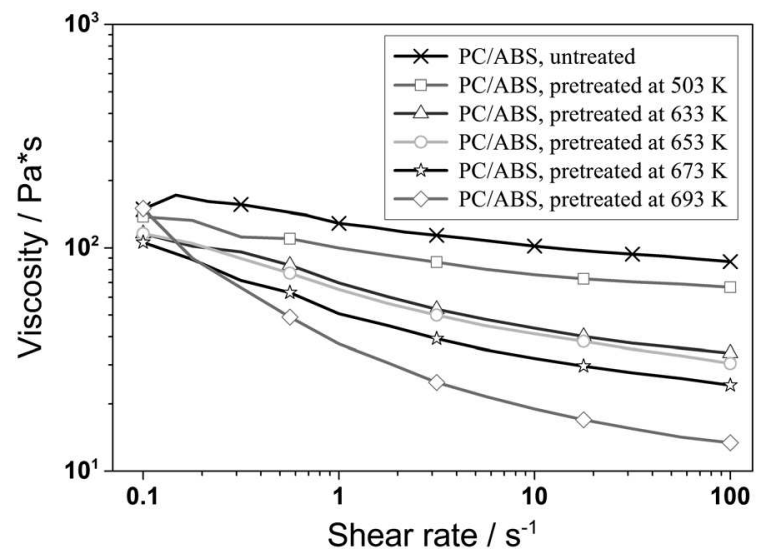

Figure 5. Viscosity of PC/ABS as a function of shear rate measured at $588 \mathrm{~K}$. 
particular at high shear rates, the thermal pretreatment leads to a decrease in viscosity of up to one order of magnitude. At low shear rates, the differences to the untreated sample are clearly below a factor of 2 and thus much less significant. It is approximated that at low shear rates the viscosity of PC/ABS is not significantly dependent on the stage of decomposition. This assessment is different from the approach and results Butler et al. proposed for polypropylene [14], where degraded and dripped material was collected and showed reduced viscosity, neither does it correspond to the recent results of Kandola et al. presented for dripping under thermo-oxidative degradation [28, 29]. Nevertheless, it is a reasonable approximation for the investigated PC/ABS blends focussing on low shear rates and pyrolysis, particularly since the respective dependencies of the viscosity on temperature and the additives used are much more pronounced.

Since dripping is associated with low shear rates, the viscosity values used for the numerical investigations were taken at the lowest shear rate measured. The lowest shear rate measured was of $0.1 \mathrm{~Hz}$. To keep the material from decomposing, the highest temperature applied in the experiments was $588 \mathrm{~K}$, sufficiently below the decomposition temperature of $600 \mathrm{~K}$. The viscosity values for higher temperatures were estimated based on the exponential fit of the isothermal measurements at $0.1 \mathrm{~Hz}$.

Figure 6 shows the data points and the estimated characteristic for higher temperatures (lines).

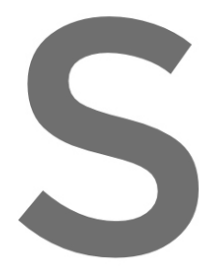

Further material properties used for modelling

The simulations focussed on the interaction of dripping behaviour, gasification and combustion of the materials in the vertical UL 94 scenario. Thus, the determination of the model input parameters concentrated on the characteristics describing the main differences between the materials. The most important difference between them are their rheologieal characteristies (see-paragraph 4.2), the char yield and the combustion effidiercy, as reported in [17]. All other
were kept the same for all fout materials.
The values for the density at room emperature were taken fron
commercial PC/ABS blend from Bayer MaterialScience. The influence
For all four materials, at density of $1130 \mathrm{~kg} \mathrm{~m}^{-3}$, a thermal conductivive
specific heat capacity of $1740 \mathrm{~J} \mathrm{~kg}^{-1} \mathrm{~K}^{-1}$ were applied. Perfect emissiv

The implementation of gasification effects in PFEM is based on a single-step first-order Arrhenius

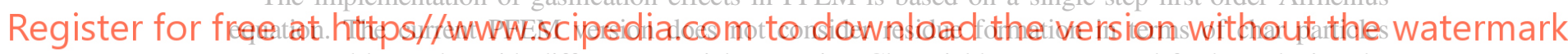
represented by nodes with different material properties. Char yield was accounted for by reducing the amount of mass loss accordingly. The decomposition kinetics of the materials was evaluated from thermogravimetric measurements. The Arrhenius coefficient A and activation energy $E$ were obtained by fitting the reaction rate to a value of $0.01 \mathrm{~s}^{-1}$ at the temperature of maximum mass loss [30]. Values of $1.4 \mathrm{e} 10 \mathrm{~s}^{-1}$ for A and $185 \mathrm{~J} \mathrm{~mol}^{-1}$ for $E$ were used in the simulations.

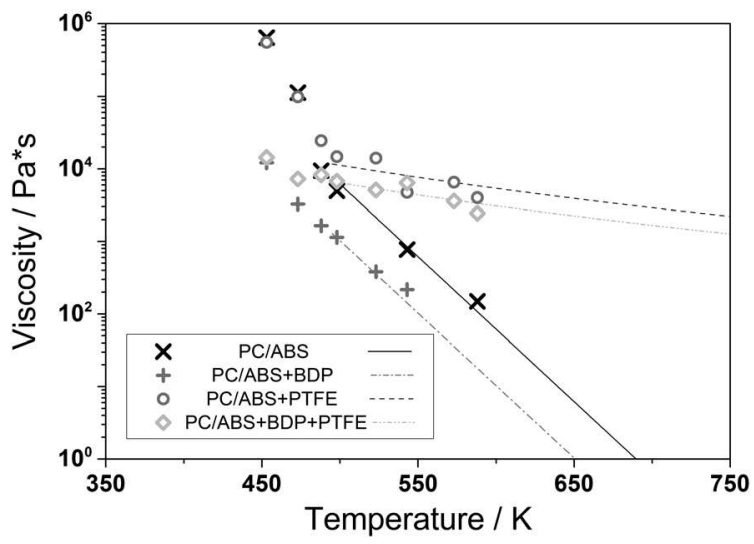

Figure 6. Rheological characteristics of the materials measured at $0.1 \mathrm{~Hz}$ under different temperatures (points) and the exponential fits (lines) used for the simulations. 
The heat of decomposition of PC/ABS was determined with DSC according to [31]. The value of $1050 \mathrm{~kJ} \mathrm{~kg}^{-1}$ was set for all materials. The heat of complete combustion of the volatiles was determined with pyrolysis combustion flow calorimetry [32]. The values of all materials differed by less than $5 \%$, and thus, the value of $27.4 \mathrm{~kJ} \mathrm{~g}^{-1}$ was used for the simulations for all materials. The char yields and the combustion efficiencies were calculated from the cone calorimeter results at an irradiation of $70 \mathrm{~kW} \mathrm{~m}{ }^{-2}$ in [17]. The combustion efficiency of PC/ABS was set to 1 . The input parameters used are summarised in Table II.

\subsection{Numerical investigation in $2 D$ : dripping}

In this first set of simulations, the submodel for thermal decomposition was turned off in order to assess the influence of the viscosity individually. The heat flux boundary condition to the sample was held constant, and no feedback was realised from the flame. Since no gasification occurs, the maximum temperatures at the surface reached temperatures clearly above the decomposition temperature. Dripping samples showed temperatures up to $850 \mathrm{~K}$, whereas $\sim 1250 \mathrm{~K}$ was observed for nondripping samples. Figure 7 shows images of the PFEM calculations simulating PC/ABS + BDP (a) and PC/ABS + PTFE (b). The images were taken between $0 \mathrm{~s}$ and $60 \mathrm{~s}$. The difference in viscosity of the two materials leads to dripping in the case of PC/ABS + BDP and prevents dripping in the calculations of PC/ABS + PTFE. The images show that the viscosity functions applied enables PFEM to reproduce the basic dripping behaviour of the materials.

The mass loss curves characteristic of all four materials in the simulations without thermal decomposition is shown in Figure 8. The materials containing PTFE do not drip and hence do not lose
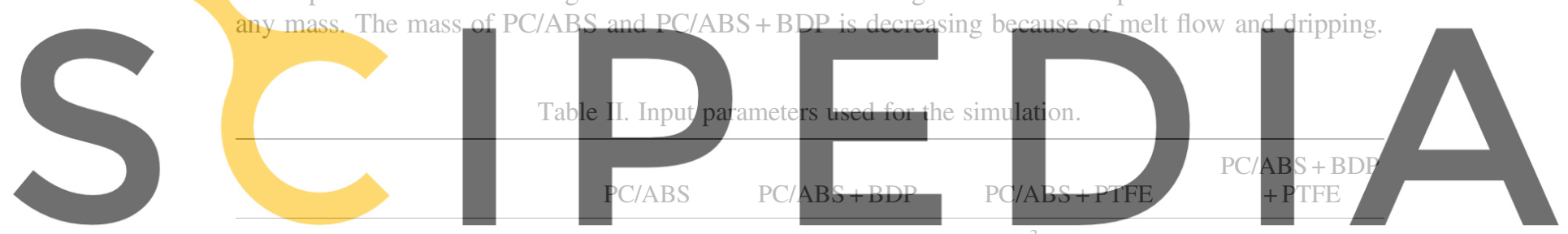

\section{Density}

Thermal conductivity

Register for freeiathattpskikwww.scipedia.com to download theiversion without the watermark

Arrhenius coefficient

Activation energy

Heat of complete combustion of the volatiles

Char yield

Combustion efficiency

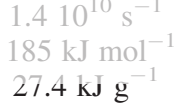

$185 \mathrm{~kJ} \mathrm{~mol}$
$27.4 \mathrm{~kJ} \mathrm{~g}$ 0.2

1
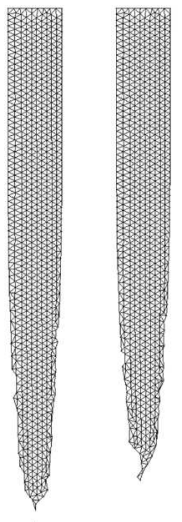

b)
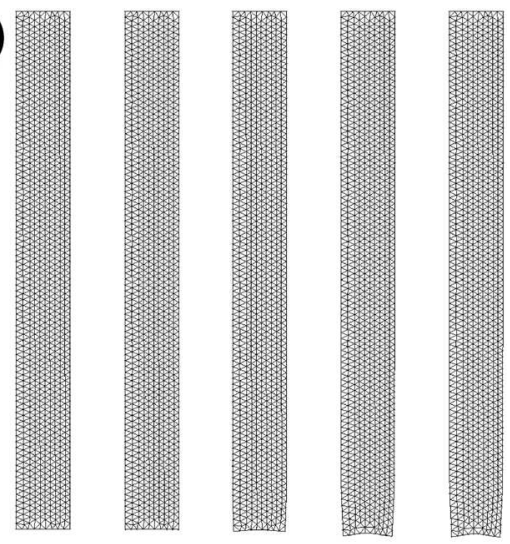
0.75

Figure 7. Images of the PFEM 2D simulations without gasification submodel for (a) PC/ABS + BDP and (b) $\mathrm{PC} / \mathrm{ABS}+\mathrm{PTFE}$ taken between $0 \mathrm{~s}$ and $60 \mathrm{~s}$. 


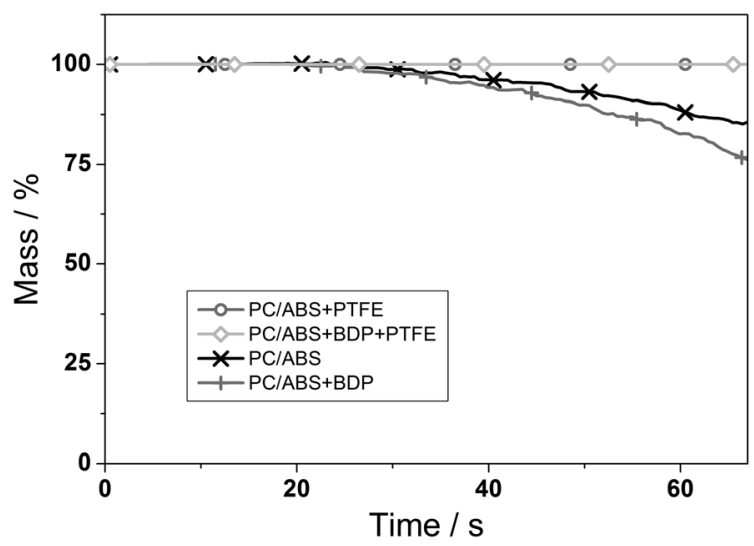

Figure 8. Mass characteristics calculated with PFEM: 2D simulations without submodel for gasification.

The dripping behaviour is more pronounced for PC/ABS + BDP due to the plasticising effect of BDP, leading to an enhanced mass loss.

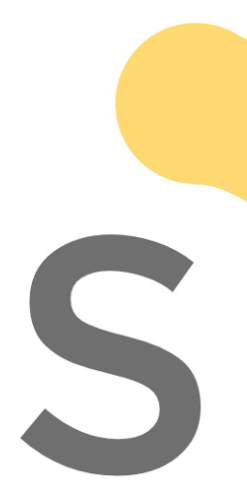

(2.5. Numerical in

4.5. Numerical investigation in 2D: dripping and gasification

In Figure 9, PFEM simulations of PC/ABS and PC/ABS + PTFE are shown. The calculations include the submodel for thermal decomposition, which consumes both mass and energy when the material is heated up. The heat flux boundary condition to the sample was held constant, and no feedback was realised from the flame. The maxinum temperatures predicted are between $800 \mathrm{~K}$ and $840 \mathrm{~K}$. They appear to be higher for PC/ABS + PTFE, because for PC/ABS the parts with the highest temperatures exhibit the lowest viscosity and drip first.

Besides the lower temperature profile, there is another impact of thermal decomposition is related to the materials te

of the sample and therefore the mass loss rate due to gasification as well. The phenomenon of removing

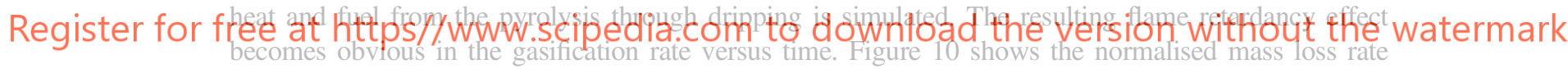
due to gasification only (gasification rate) over time for all four materials. With time, the samples heat up until thermal decomposition starts and progresses. Between $20 \mathrm{~s}$ and $30 \mathrm{~s}$, the gasincation rate of the nondripping materials PC/ABS $+\mathrm{PTFE}$ and PC/ABS $+\mathrm{BDP}+\mathrm{PTFE}$ reaches a plateau. Subsequently, the samples decompose at a constant rate. The gasification rates of the dripping samples PC/ABS and $\mathrm{PC} / \mathrm{ABS}+\mathrm{BDP}$ have a different shape. When dripping starts, which takes place earlier for PC/ $\mathrm{ABS}+\mathrm{BDP}$ than for $\mathrm{PC} / \mathrm{ABS}$, the gasification rate decreases compared to the nondripping samples. In the UL 94 test, the competing effects of gasification and dripping are crucial in determining the
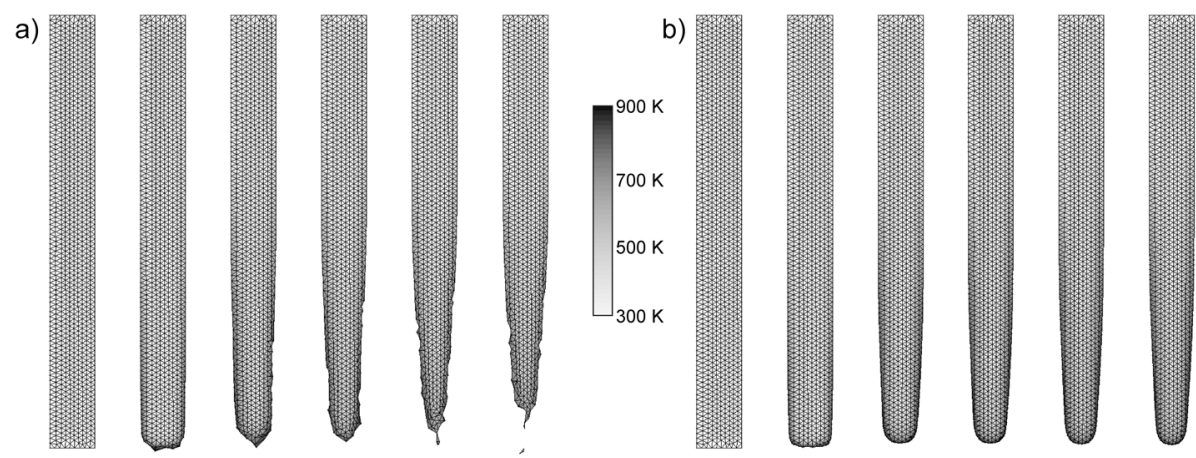

Figure 9. Images of the PFEM 2D simulations, including the submodel for gasification, for (a) PC/ABS and (b) PC/ABS + PTFE taken between $0 \mathrm{~s}$ and $60 \mathrm{~s}$. 


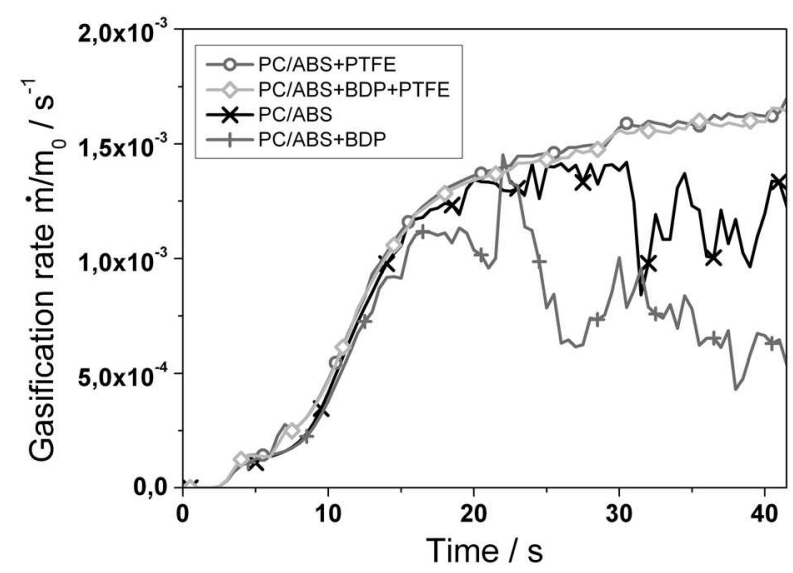

Figure 10. Gasification rate over time simulated with 2D-PFEM under constant external heat flux.

material's behaviour, therefore affecting the classification. Figure 10 indicates that PFEM is able to predict the competing effects of gasification and dripping.

\subsection{Numerical investigation in 3D: dripping and gasification}

For the three-dimensional simulations, the input parameters were kept the same as for the two-dimensional ones. The submodel for thermal decomposition was included. The boundary conditions of the sample changed as a result of the change in dimensionality. Instead of the heat load per unit length of the edges in the 2D problem, in 3D all sides were heated up according to the UL 94 experiment. Figure 11 illustrates the 3D simulations of the dripping materials PC/ABS and PC/ABS + BDP. The images were taken every $2 \mathrm{~s}$ until substantial dripping occurred. Maximum surface temperatures were in the same range as for $2 \mathrm{D}$ calculations, around $820 \mathrm{~K}$, because of the same values used for Arrhenius coefficient, activation energy and the heat of decomposition.

Under the heating conditions applied for this set of simulations, PFEM predicted substantial dripping for the PC/ABS sample to take place after $\sim 12 \mathrm{~s}$. This value is in the range of the average value for the sum of ignition plus burning time observed in the UL 94 test of PC/ABS (17.7 s), which extinguished by dripping. For PC/ABS + BDP, PFEM predicted substantial dripping taking place $\sim 4 \mathrm{~s}$ earlier. In the UL 94 tests of $\mathrm{PC} / \mathrm{ABS}+\mathrm{BDP}$, the average sum of ignition plus burning time was $13.3 \mathrm{~s}$. The dripping of the samples was not only modelled well with respect to the principal phenomena but also in good accordance with experimental results.

The gasification rates shown in Figure 12 indicate that for the nondripping materials PC/ABS + PTFE and PC/ABS + BDP + PTFE, a plateau is reached after $10 \mathrm{~s}$ to $15 \mathrm{~s}$. As a result of dripping, the gasification rate of $\mathrm{PC} / \mathrm{ABS}$ and $\mathrm{PC} / \mathrm{ABS}+\mathrm{BDP}$ is decreased. Substantial dripping is highlighted by the strong decrease in the gasification rate. The times until substantial dripping correspond to the times determined

a)

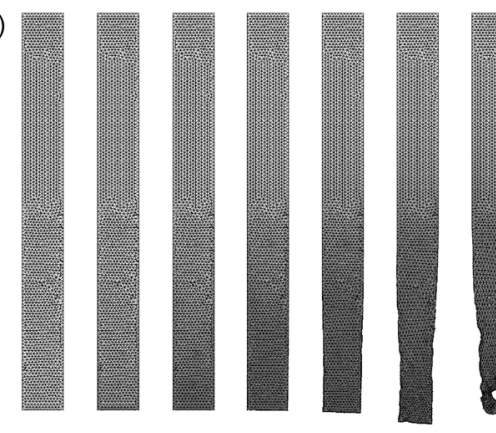

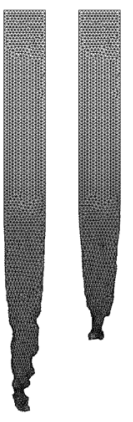

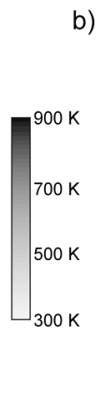

b)

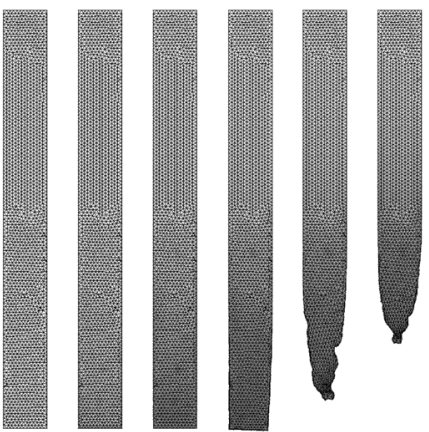

Figure 11. Images of three-dimensional PFEM simulations for (a) PC/ABS and (b) PC/ABS + BDP taken every $2 \mathrm{~s}$. 


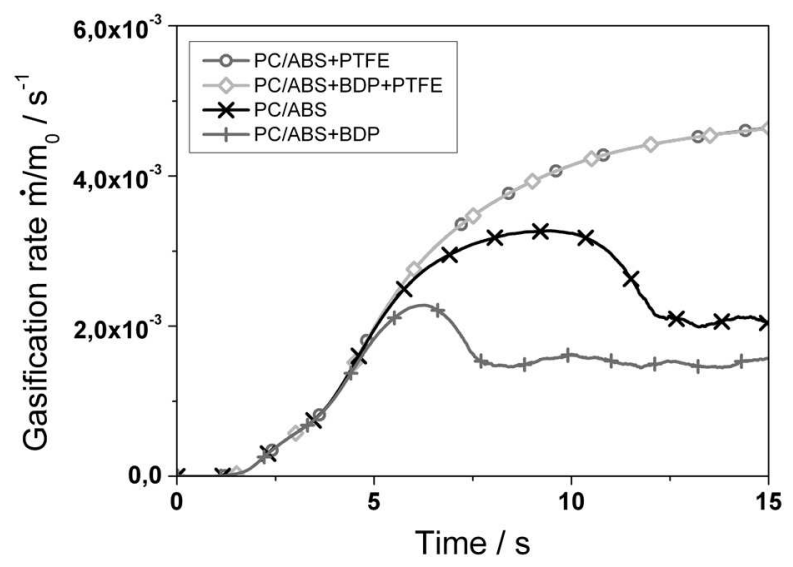

Figure 12. Gasification rate over time simulated with 3D-PFEM under constant external heat flux.

from the images in Figure 11. The gasification rates of the dripping materials become steady after dripping starts. In this region, dripping and gasification take place continuously, due to the constant heat flux applied to the sample. The plateau of the gasification rate is higher for the material PC/ABS, because dripping occurs more slowly than for PC/ABS + BDP, and hence, temperatures and gasification rate are increased. The simulation covers the interaction and competition of the two phenomena dripping and gasification well. The plasticisation effect of BDP is covered as well as the time scale.

\subsection{Numerical investigation in 3D: dripping, gasification and combustion}

In order to account for the flame retardant characteristics of BDP in the simulations, a simple combustion submodel has been implemented. The impact of BDP and the differences in the fire behaviour of the blends were modelled by adjusting the combustion efficiency and the char yield of the samples as described before. Compared to PC/ABS, the simulated heat release rate was reduced by $6 \%$ for $\mathrm{PC} /$ $\mathrm{ABS}+\mathrm{PTFE}, 23 \%$ for PC/ABS + BDP and $34 \%$ for PC/ABS + BDP + PTFE, according to the fire behaviour reported in [17]. Figure 13 presents the gasification rate over time of the simulations including dripping, gasification and the simple combustion model, taking into account flame inhibition and charring through constant reducing factors that correspond to the reduced combustion efficiency and 1 minus char yield, respectively. The heat boundary condition of the samples for $0 \mathrm{~s}<t<10 \mathrm{~s}$ consists of the ignition source (applied burner in the UL 94 test) and the heat feedback of the combustion model. For $t>10 \mathrm{~s}$, the external heat flux to the sample is a result of the simple combustion model.

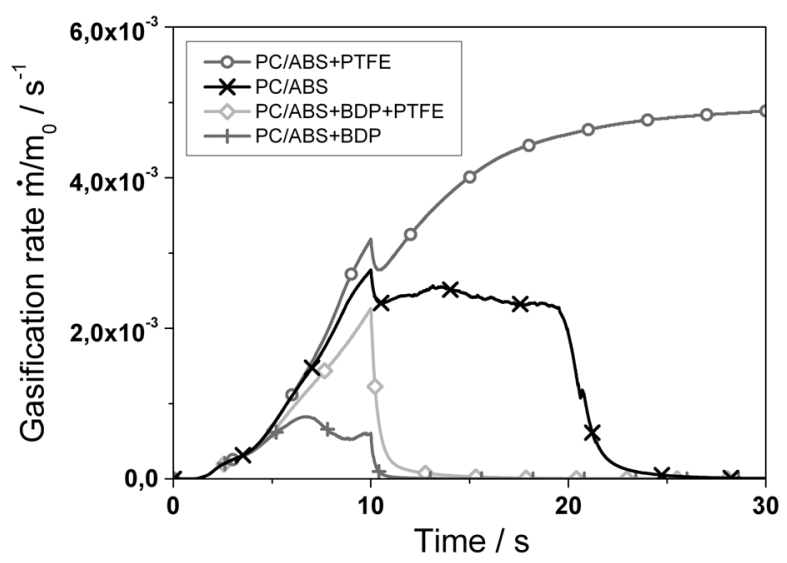

Figure 13. Gasification rate over time simulated with 3D-PFEM: heat feedback from ignition source (for $0<$ $t<10$ ) and simplified combustion model (for $t>0$ ). 
For the first $5 \mathrm{~s}$, the graphs run in parallel, because the same ignition source effect is dominant and thermal properties are the same for all samples. The graphs begin to diverge as the effect of the dripping behaviour and the combustion characteristics increases. At $t=10 \mathrm{~s}, \mathrm{PC} / \mathrm{ABS}+\mathrm{PTFE}$ shows the highest gasification rate. As melt flow/dripping begins, the gasification rate of PC/ABS is slightly reduced. For PC/ABS + BDP + PTFE, the changed combustion characteristics (reduced combustion efficiency due to flame inhibition and reduced amount of fuel due to charring) are the reason for the decreased thermal decomposition. The gasification rate of PC/ABS + BDP is reduced substantially due to both enhanced dripping behaviour and less heat feedback from the combustion model.

When the ignition source is turned off $(t>10 \mathrm{~s})$, the model predicts for PC/ABS + PTFE that even though the gasification rate drops first, it remains high enough to maintain and enhance the burning of the sample. The gasification rate of PC/ABS drops after $10 \mathrm{~s}$ to a level where dripping and gasification take place simultaneously. These unstable conditions end when substantial dripping reduces the gasification rate until burning is no longer maintained. The prediction of such sensitive unstable behaviour resulting in a significant uncertainty in time-to-extinction corresponds well with the experimental results. Also in the experimental determination of the UL 94 classification for PC/ABS, the specimens show a significant variation in burning time, since each drip nearly leads to extinction until a final drip achieves it. For PC/ABS + BDP and PC/ABS + BDP + PTFE, the heat feedback to the sample due to combustion is too small to sustain thermal decomposition and the samples extinguish quickly $(<2 \mathrm{~s})$ after the ignition source is turned off. The complex behaviour and the resulting performance of the four materials were modelled in good accordance with the experimental results.

Figure 13 demonstrates the ability of PFEM to model the competing effects of dripping and gasification as well as their interaction with each other. Furthermore, flame inhibition and charring is taken into account reasonably in a simple model for the combustion and the resulting thermal feedback from the flame in the UL 94 scenario. The UL 94 classification of the four blends is predicted in good accordance with the experimental results. PC/ABS + PTFE burns completely (HB), PC/ABS and PC/ ABS + BDP extinguish by dripping (V-2) and PC/ABS + BDP + PTFE extinguishes almost immediately without dripping $(\mathrm{V}-0)$. What is more, not only the principal phenomena and classification but also the time scales are simulated extremely well, even though substantial assumptions and simplifications have been made. The pyrolysis submodel is based on simple, thermally activated, single-step decomposition. Although the dependency of the main mass loss on temperature and heating rate is described rather well, such an approximation usually fails to describe the beginning and the end of decomposition. As in other simulations of the fire behaviour of polymers [30,33, 34], it seems that using such a simple model is good enough to model the pyrolysis during burning. The formation of char particles as well as residual protection layers was not modelled at all. The fact that modelling the reduction of fuel by charring was enough to obtain a satisfactory description of the behaviour may be a property of the chosen material and perhaps different for other materials. The burner as well as the heat feedback on the sample due to combustion is implemented into the model in a simple and reasonable way but is arbitrary to some extent. This aspect of the presented simulation requires further development in the future. Advances regarding the determination of boundary conditions and material properties as a function of temperature are also needed to enable further improvement of the model. Nevertheless, this work presents a successful approach for numerically modelling the complex behaviour of polymers in the UL 94 test including the essential aspects of dripping, gasification and combustion in both a qualitative and a quantitative manner.

\section{CONCLUSIONS}

The fire behaviour of four PC/ABS blends in the UL 94 test was studied both experimentally and numerically. The focus was on the impact of the additives BDP and PTFE on the dripping behaviour of PC/ABS. The material system covered different UL 94 classifications (V-0; V-2; HB) as a result of the dripping and combustion characteristics.

The materials were characterised thoroughly, particularly with regard to their rheological properties. The addition of BDP decreased the viscosity of PC/ABS due to the plasticiser effect of BDP. Adding PTFE led to the formation of a flow limit and increased viscosity at low shear rates. The rheological 
assumptions explained the different fire behaviour in the UL 94. Dripping was observed for PC/ABS and was even more pronounced for PC/ABS + BDP. The materials containing PTFE did not drip at all.

The numerical investigation was conducted with the particle finite element method (PFEM). The current version of the method accounts for large deformations in the fluid-free surface, thermal decomposition of the material and changes in viscosity as a function of temperature. Additionally, a simple combustion model to simulate the heat feedback of the flame to the pyrolyzing sample was implemented, taking flame inhibition and charring into account. The heat release was reduced by factors representing the reduced combustion efficiency, and the fuel release reduced according to the char yield. The temperature-dependent values determined for the viscosity of the investigated materials enabled PFEM to predict the dripping and nondripping behaviour of the different materials correctly. PFEM identified the competition of dripping and gasification as well as the interaction between both phenomena. The main impact of flame inhibition and charring was also simulated well for the chosen systems. From the simulations, the main phenomena, the different UL 94 classification of the materials and the times to extinction were derived in accordance with the experimental results.

The numerical investigation indicated the potential of PFEM to model polymer-related fire problems like the UL 94 scenario. The performed PFEM simulations have the ability to investigate the competition and interaction between different main phenomena controlling the fire such as dripping, gasification, charring, combustion and flame inhibition. Thus, this and following studies increase our understanding of the complex behaviour of polymeric materials during UL 94 testing. Further, the PFEM simulation based on reasonable assumptions and advanced determination of some important input parameter delivered a rather perfect description of the performance without any directed fitting or adjusting. Subject to validation also a prediction of UL 94 performance is possible.

\section{ACKNOWLEDGEMENTS}

The authors thank T. Himmel from the Berlin Institute of Technology for conducting the rheological investigations and Prof. Dr.-Ing. M. Wagner for supporting this cooperation.

\section{REFERENCES}

1. Zhang J, Shields TJ, Silcock GWH. Effect of melting behavior on upward flame spread of thermoplastics. Fire and Materials 1997; 21:1-6.

2. Zhang J, Shields TJ, Silcock GWH. Fire hazard assessment of polypropylene wall linings subjected to small ignition sources. Journal of Fire Sciences 1996; 14:67-84.

3. Sherrat J, Drysdale D. The effect of the melt-flow process on the fire behaviour of thermoplastics. In Interflam 2001. Interscience Communications: London, 2001; 149-159.

4. Ohlemiller TJ, Butler KM. Influence of polymer melt behaviour on flammability. Proceedings of the 15th Joint Panel Meeting on Fire Research and Safety, UJNR, Volume 1, 2000; 81-88.

5. Denecker C, Liggat JJ, Snape CE. Relationship between the thermal degradation chemistry and flammability of commercial flexible polyurethane foams. Journal of Applied Polymer Science 2006; 100:3024-3033.

6. IEC 60695-11-10. Fire hazard testing - part 11-10: test flames - 50W horizontal and vertical flame test methods, 1999.

7. Schartel B. Uses of fire tests in materials flammability development. In Fire retardancy of polymeric materials (2nd edn), Morgan AB, Wilkie CA (eds). CRC Press: Boca Raton, 2010, chap. 15; 387-420.

8. Wang Y, Zhang F, Chen X, Jin Y, Zhang J. Burning and dripping behaviors of polymers under UL 94 vertical burning test conditions. Fire and Materials 2010; 34:203-215.

9. Wang Y, Zhang J. Influences of specimen size and heating mode on the ignitability of polymeric materials in typical small-scale fire test conditions. Fire and Materials 2011; 36:231-240.

10. Wang Y, Zhang F, Jiao C, Jin Y, Zhang J. Convective heat transfer of the Bunsen flame in the UL94 vertical burning test for polymers. Journal of Fire Sciences 2010; 28:337-356.

11. Wang Y, Zhang J, Jow J, Su K. Analysis and modeling of ignitability of polymers in the UL-94 vertical burning test condition. Journal of Fire Sciences 2009; 27:561-581.

12. Quintiere JG, Downey BP, Lyon RE. An investigation of the vertical Bunsen burner test for flammability of plastics. Report No. DOT/FAA/AR-TN11/19, US Department of Transport, 2012.

13. Butler KM, Ohlemiller TJ, Linteris GT. A progress report on numerical modeling of experimental polymer melt flow behavior. In Interflam 2004. Interscience Communications: London, 2004; 937-948.

14. Butler KM, Oñate E, Idelsohn SR, Rossi R. Modeling polymer melt flow using the particle finite element method. In Interflam 2007, vol. 2. Interscience Communications: London, 2007; 929-940.

15. Oñate E, Rossi R, Idelsohn SR, Butler KM. Melting and spread of polymers in fire with the particle finite element method. International Journal for Numerical Methods in Engineering 2009; 81:1046-1072.

16. www.cimne.com/pfem 19 June 2014. 
17. Pawlowski KH, Schartel B. Flame retardancy mechanisms of triphenyl phosphate, resorcinol bis(diphenyl phosphate) and bisphenol A bis(diphenyl phosphate) in polycarbonate/ acrylonitrile-butadiene-styrene blends. Polymer International 2007; 56:1404-1414.

18. Oñate E, Idelsohn SR, Del Pin F, Aubry R. The particle finite element method. An overview. International Journal of Computational Methods 2004; 1:267-307.

19. Idelsohn SR, Oñate E, Del Pin F. A Lagrangian meshless finite element method applied to fluid-structure interaction problems. Computers and Structures 2003; 81:655-671.

20. Idelsohn SR, Oñate E, Del Pin F. The particle finite element method: a powerful tool to solve incompressible flows with free-surfaces and breaking waves. International Journal for Numerical Methods in Engineering 2004; 61:964-989.

21. Oñate E, Idelsohn SR, Celigueta MA, Rossi R. Advances in the particle finite element method for the analysis of fluid-multibody interaction and bed erosion in free surface flows. Computer Methods in Applied Mechanics and Engineering 2008; 197:1777-1800.

22. Idelsohn SR, Marti J, Limache A, Oñate E. Unified Lagrangian formulation for elastic solids and incompressible fluids: Application to fluid-structure interaction problems via the PFEM. Computer Methods in Applied Mechanics and Engineering 2008; 197:1762-1776.

23. Idelsohn SR, Marti J, Souto-Iglesias A, Oñate E. Interaction between an elastic structure and free-surface flows: experimental versus numerical comparisons using the PFEM. Computational Mechanics 2008; 43:125-132.

24. Idelsohn SR, Oñate E, Calvo N, Del Pin F. The meshless finite element method. International Journal for Numerical Methods in Engineering 2003; 58:893-912.

25. Hamins A, Bundy M, Dillon SE. Characterization of candle flames. Journal of Fire Protection Engineering 2005; 15:265-285.

26. Perret B, Pawlowski KH, Schartel B. Fire retardancy mechanisms of arylphosphates in polycarbonate(PC) and PC/acrylonitrile-butadiene-styrene: the key role of decomposition temperature. Journal of Thermal Analysis and Calorimetry 2009; 97:949-958.

27. Wawrzyn E, Schartel B, Ciesielski M, Kretzschmar B, Braun U, Döring M. Are novel aryl phosphates competitors for bisphenol A bis(diphenyl phosphate) in halogen-free flame-retarded polycarbonate/acrylonitrile-butadiene-styrene blend? European Polymer Journal 2012; 48:1561-1574.

28. Kandola B, Price D, Milnes J, DaSilva A. Development of a novel experimental technique for quantitative study of melt dripping of themoplastic polymers. Polymer Degradation and Stability 2013; 98:52-63.

29. Kandola BK, Price D, Milnes GJ, Silva A, Gao F, Nigmatullin R. Characterization of melt dripping behavior of flame retarded polypropylene nanocomposites. In Fire and polymers VI: new advances in flame retardant chemistry and science, Morgan AB, Wilkie CA, Nelson GL (eds), vol. 1118. ACS Symposium Series; Washington, 2012, chap. 21; 311-325.

30. Stoliarov SI, Safronava N, Lyon RE. The effect of variation in polymer properties on the rate of burning. Fire and Materials 2009; 33:257-271.

31. Stoliarov SI, Walters RN. Determination of the heats of gasification of polymers using differential scanning calorimetry. Polymer Degradation and Stability 2008; 93:422-427.

32. Schartel B, Pawlowski KH, Lyon RE. Pyrolysis combustion flow calorimeter: a tool to assess flame retarded PC/ABS materials? Thermochimica Acta 2007; 462:1-14.

33. Kempel F, Schartel B, Linteris GT, Stoliarov SI, Lyon RE, Walters RN, Hofmann A. Prediction of the mass loss rate of polymer materials: impact of residue formation. Combustion and Flame 2012; 159:2974-2984.

34. Mouritz AP, Gibson AG. Fire properties of polymer composite materials. Springer: Dordrecht, 2006. 